ARTICLE

Received 10 Nov 2013 | Accepted 15 Jul 2014 | Published 18 Aug 2014 DOI: 10.1038/ncomms5693

\title{
Interface-induced nonswitchable domains in ferroelectric thin films
}

\author{
Myung-Geun Han ${ }^{1}$, Matthew S.J. Marshall ${ }^{2}$, Lijun Wu', Marvin A. Schofield', Toshihiro Aoki ${ }^{3}$,
} Ray Twesten ${ }^{4}$, Jason Hoffman², Frederick J. Walker², Charles H. Ahn² \& Yimei Zhu ${ }^{1}$

Engineering domains in ferroelectric thin films is crucial for realizing technological applications including non-volatile data storage and solar energy harvesting. Size and shape of domains strongly depend on the electrical and mechanical boundary conditions. Here we report the origin of nonswitchable polarization under external bias that leads to energetically unfavourable head-to-head domain walls in as-grown epitaxial $\mathrm{PbZr}_{0.2} \mathrm{Ti}_{0.8} \mathrm{O}_{3}$ thin films. By mapping electrostatic potentials and electric fields using off-axis electron holography and electron-beam-induced current with in situ electrical biasing in a transmission electron microscope, we show that electronic band bending across film/substrate interfaces locks local polarization direction and further produces unidirectional biasing fields, inducing nonswitchable domains near the interface. Presence of oxygen vacancies near the film surface, as revealed by electron-energy loss spectroscopy, stabilizes the charged domain walls. The formation of charged domain walls and nonswitchable domains reported in this study can be an origin for imprint and retention loss in ferroelectric thin films.

\footnotetext{
${ }^{1}$ Condensed Matter Physics and Materials Science, Brookhaven National Laboratory, Upton, New York 11973, USA. ${ }^{2}$ Department of Applied Physics and Center for Research on Interface Structures and Phenomena, Yale University, New Haven, Connecticut 06520, USA. ${ }^{3}$ JEOL USA Inc., Peabody,

Massachusetts 01960, USA. ${ }^{4}$ Gatan Inc., Pleasanton, California 94588, USA. Correspondence and requests for materials should be addressed to M.-G.H. (email: mghan@bnl.gov) or to Y.Z. (email: zhu@bnl.gov).
} 
O ne method of realizing a non-volatile logic element ${ }^{1}$ is to use a ferroelectric material to induce a charge-mediated change of state in an adjacent material. A ferroelectric has a spontaneous electrical polarization that is switchable upon the application of a DC voltage $e^{2,3}$. However, a major issue for commercialization has been the poor reliability of ferroelectric memory devices including a decrease in polarization with repeated switching (fatigue), polarization backswitching leading to loss of data (retention loss) and preferential polarization state (imprint) $)^{3-7}$. Ground-state domain structures and switching processes in ferroelectric thin films are difficult to predict because of the importance of electrical and mechanical boundary conditions present at the film interfaces ${ }^{8}$. The primary mechanical boundary condition for an epitaxial thin film is the strain that results from the lattice mismatch between the film and substrate $e^{8-11}$. Electrical boundary conditions can dramatically influence ground-state domain structures and switching via polarization charge compensation mechanisms at the interfaces ${ }^{12,13}$, the formation of $180^{\circ}$ stripe domains ${ }^{14}$, fluxclosure domain structures ${ }^{15,16}$ and interfacial reconstructions ${ }^{17}$. Mobile charge carriers arising from vacancies and impurities also play an important role by internally compensating the polarization charges or by modifying the electric field within the ferroelectric in response to an external bias ${ }^{17-20}$. In addition, achieving chemical potential equilibrium between ferroelectric thin films and electrode materials can cause the accumulation or depletion of mobile charge carriers, thereby leading to built-in potentials at the interfaces ${ }^{18,19,21}$.

The macroscopic ferroelectric domain structures and their switching properties can be understood by directly visualizing domains during switching and characterizing the electrostatic potentials. Here, we report the unusual formation of charged $180^{\circ}$ domain walls in head-to-head configuration in ferroelectric epitaxial thin films observed by dark-field transmission electron microscopy (TEM) combined with in situ electrical biasing ${ }^{22-26}$. Electrostatic potential and electric field distributions within ferroelectric films under external biases were, for the first time in our knowledge, mapped out using two techniques: off-axis electron holography ${ }^{27}$ and electron-beam-induced current (EBIC) measurement ${ }^{28}$. We found that electronic band bending across the film/substrate interfaces pins the local polarization direction and produces unidirectional biasing fields, inhibiting local domain switching and leading to formation of charged $180^{\circ}$ domain walls in an unstable head-to-head configuration. Surprisingly, these positively charged domain walls were found to be stable over several months. Our electron-energy loss spectroscopy data revealed higher concentration of oxygen vacancies near the film surface, which implies internal charge compensation by mobile charge carriers.

\section{Results}

Characterization of domain structures. Epitaxial $\mathrm{PbZr}_{0.2} \mathrm{Ti}_{0.8} \mathrm{O}_{3}$ (001) (PZT) thin films were grown on 0.5 wt.\% Nb-doped $\mathrm{SrTiO}_{3}$ (001) (Nb-STO) by off-axis radiofrequency magnetron sputtering at $515^{\circ} \mathrm{C}$ and cooled to room temperature in $225 \mathrm{mTorr}$ of process gases. To facilitate in situ electrical biasing of the PZT films, a capacitor geometry with conventional Pt electrodes is fabricated using focused-ion beam (FIB) after Au sputtering, as shown in Fig. 1a (ref. 27). A probe electrical contact in the TEM holder (Nanofactory Instruments $A B$ ) is made to the top electrode in situ in TEM, while the counter electrode is connected to the conducting $\mathrm{Nb}$-STO substrate using silver paste. To maximize contrast associated with $180^{\circ}$ ferroelectric domains because of violation of Friedel's law ${ }^{29,30}$, samples were tilted from the [010] zone axis for a two-beam condition and dark-field images were obtained with series of external biases
(Figs $1 \mathrm{a}$ and $2 \mathrm{a}-\mathrm{e}$ ). Owing to $\sim 1.2 \%$ biaxial compressive strain, the PZT thin films consist primarily of domains with out-of-plane polarization $^{31}$, although they are likely fully relaxed because of the presence of misfit dislocations near the interface. The $c$ domains in as-grown PZT thin films have the polarization direction, either upwards or downwards, which are henceforth referred to as $P_{\text {up }}$ or $P_{\text {down }}$ domains, respectively. Conventionally, stripe patterns with alternating polarization direction have been predicted theoretically as a ground domain structure and experimentally observed for ferroelectric thin films ${ }^{1}$. However, we observed the domain structure in as-grown PZT film shown in Fig. 1c showing surprisingly two domains with opposite polarizations met in the middle of a film in a head-to-head configuration. Previously, occurrence of charged domain walls, especially during polarization-switching process, has been reported from various in situ switching experiments ${ }^{22-24,32}$. However, the head-to-head domain structure observed in the as-grown PZT thin films is remarkable as such domain walls are positively charged and thus energetically unfavourable for a ground state. Furthermore, domain walls are not flat, but rather zigzag in shape, with an additional energy cost associated with this configuration. The zigzag-like domain walls can be a consequence of domain wall pinning at structural defects within the film ${ }^{33}$. Piezoresponse force microscopy (PFM) result (Fig. 1d) also supports the presence of charged domain walls embedded in PZT thin films showing an intermediate piezoresponse signal for an as-grown region compared with two oppositely poled regions with $\pm 10 \mathrm{~V}$. Note that stripe domains with alternating $180^{\circ}$ domains can be ruled out by the monotonous contrast in the PFM image. A typical current-voltage $(I-V)$ curve (Fig. 1b) taken from the TEM sample in Fig. 1a shows a rectifying property of Au top electrode/ PZT/Nb-STO structure. Similar electric transport properties have been reported for PZT films grown on Nb-STO substrates, attributed to $p n$ junction-like band bending at the film/substrate interface $^{34}$. We notice two paths of leakage currents for TEM samples prepared by FIB milling. The first contribution is from mobile charge carriers in ferroelectric films because of vacancies and impurities serving as dopants and defect-mediated conduction such as Frenkel-Poole conduction considering large concentration of structural defects associated with strain relaxation $^{3}$. The second is from FIB-milling-induced damages on the top and bottom surfaces of cross-sectioned TEM samples ${ }^{27}$. The latter can be minimized by careful FIB milling with low-energy $\mathrm{Ga}^{+}$ions. These leakage currents can be approximated as a linear resistor connected in parallel to the ferroelectric films. However, the clear rectifying behaviour shown in Fig. 1b suggests that charge transport is not controlled by the leakage. We also point out that illuminating the PZT/Nb-STO interface with the TEM electron beam generates excess charge carriers and thus increases conductivity of the film similar to optical excitations. With illumination of $200 \mathrm{kV}$ electron beam at a beam current of $\sim 1 \mathrm{nA}$, additional currents are measured for both positive and negative biases, as shown in Fig. 1b. A small open-circuit voltage $\left(V_{\mathrm{OC}}\right)$ of $-0.4 \mathrm{eV}$ is measured under electron beam illumination, as shown in the inset of Fig. 1 b.

In situ domain switching. The domain evolution under various external biases using dark-field TEM imaging combined with in situ electrical biasing is summarized in Fig. 2. Upon application of positive bias, $P_{\text {up }}$ domains (dark region) grow towards the top interface. With $3-\mathrm{V}$ bias, some $P_{\text {up }}$ domains reached the top electrode but significant areas near the top electrode are left unswitched, indicating that domain switching is highly inhomogeneous, possibly because of random domain wall pinning by structural defects. Under a negative applied bias, $P_{\text {down }}$ domains (bright region) grow towards the bottom interface. However, in 

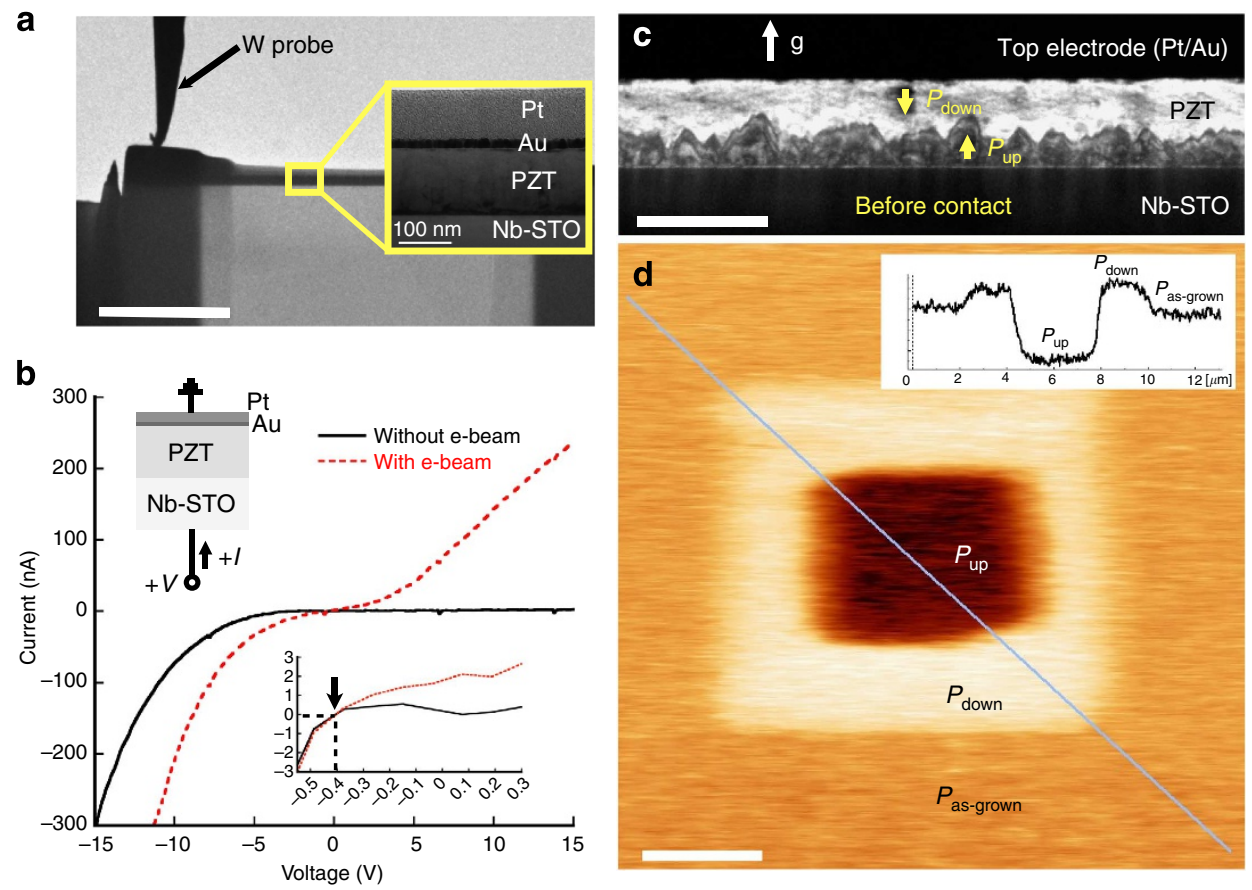

Figure 1 | Epitaxial PZT thin films on Nb-STO substrate. (a) A TEM image showing an electrical contact made for in situ biasing experiment. The scale bar is $2 \mu \mathrm{m}$. (b) A typical current-voltage $(I-V)$ curve from the TEM sample showing a clear rectifying behaviour. The inset $I-V$ shows $-0.4 V$ of $V_{\text {OC. }}$. (c) A DF TEM image with the 003 reflection of PZT lattice showing unusual head-to-head charged domain walls formed in the as-grown PZT film. The scale bar is $200 \mathrm{~nm}$. This image was taken before making an electrical contact. (d) A PFM image containing two oppositely poled states (dark contrast $P_{\text {up }}$ and bright contrast $P_{\text {down }}$ ) with external biases of $10 \mathrm{~V}$ in magnitude. The scale bar is $2 \mu \mathrm{m}$. Background is the as-grown state.
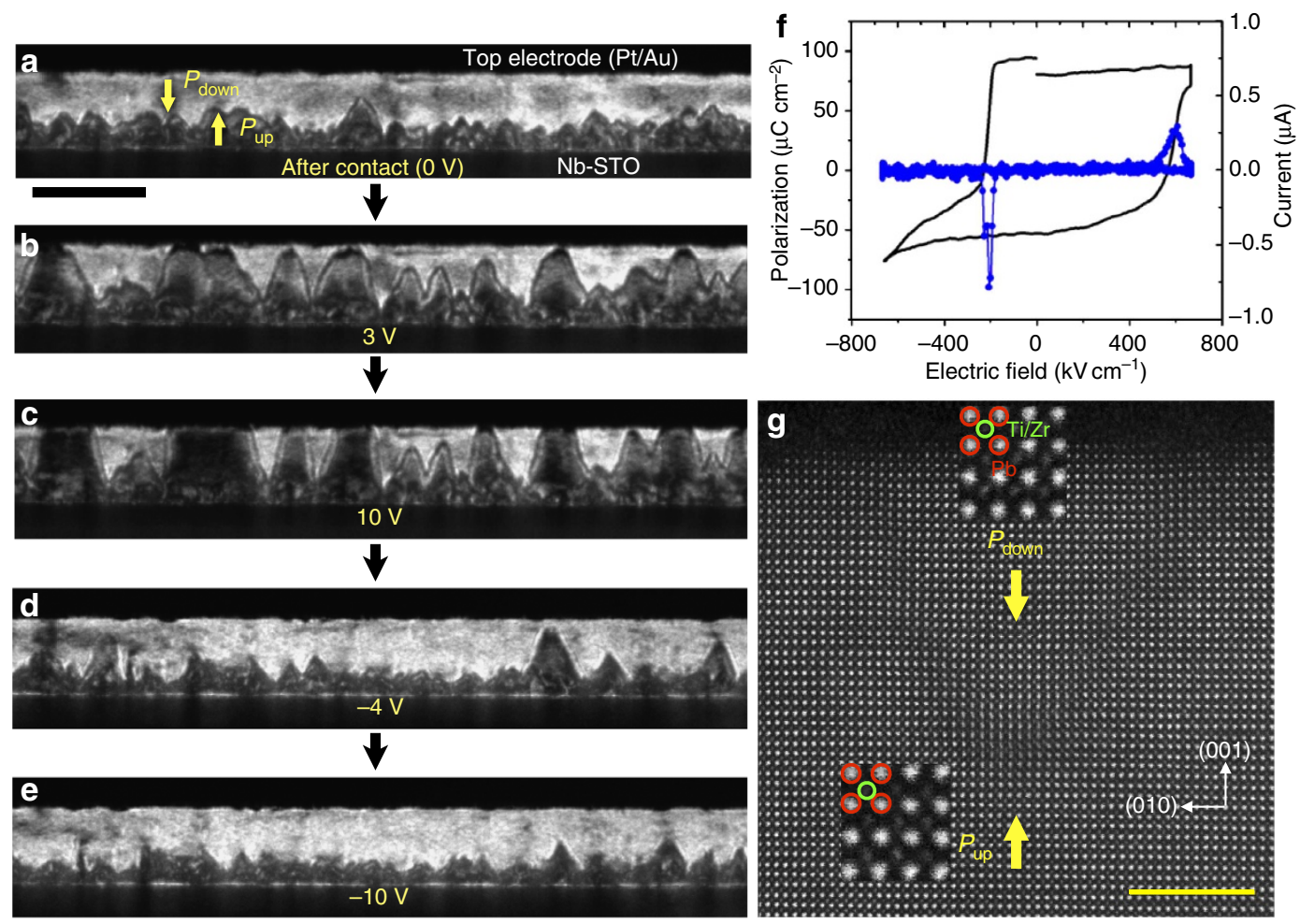

Figure 2 | Domain switching behaviour. (a-e) A series of DF TEM images showing domain-switching behaviour induced by a series of external biases. The scale bar is $200 \mathrm{~nm}$. Sequence of biasing is shown with arrows. The 0-V image (a) was taken right after making the electrical contact, which shows similar domain structure as Fig 1c. Note that domain switching do not occur near the bottom interface. (f) A P-E loop obtained from PZT thin film with switching currents (blue). (g) An atomic resolution STEM-HAADF image of a head-to-head domain configuration. The scale bar is $5 \mathrm{~nm}$. Two insets are enlarged images for each domain showing that the $\mathrm{Ti} / \mathrm{Zr}$ columns are displaced upwards (downwards) with respect to the $\mathrm{Pb}$ columns for the $P_{\text {down }}\left(P_{\text {up }}\right)$ domain. Yellow arrows indicate the polarization directions. 
this case, no $P_{\text {down }}$ domain reaches the bottom interface, resulting in the formation of charged $180^{\circ}$ domain walls in the film. Application of negative bias up to $-15 \mathrm{~V}$ (not shown here) does not result in domain switching beyond that shown in Fig. 2e. It can be concluded that switching behaviour observed in Fig. 2 is highly asymmetric ( $P_{\text {up }}$ domain is preferred) and $P_{\text {up }}$ domain near the bottom interface is not switchable. A $P$-E loop obtained from PZT thin films also shows an asymmetric switching, as shown in Fig. 2f. Here the electric fields are estimated with the PZT film thickness of $150 \mathrm{~nm}$. The remnant polarizations are about 80 and $50 \mu \mathrm{Ccm}^{-2}$ for $P_{\text {up }}$ and $P_{\text {down }}$ domains, respectively. We find that this $P-E$ loop agrees well with the TEM observations in Fig. 2a-e. The negative switching current sharply peaked near $-0.2 \mathrm{MV} \mathrm{cm}^{-1}$ agrees with rapid switching at $-4 \mathrm{~V}$ (Fig. 2d) followed by almost no additional switching with $-10 \mathrm{~V}$ (Fig. 2e). For the positive switching, a dramatic switching occurs between 0 and $3 \mathrm{~V}$ (Fig. 2b) and switching continues between $3 \mathrm{~V}$ (Fig. 2b) and $10 \mathrm{~V}$ (Fig. 2c) without completing switching, implying that the coercive field for $P_{\text {up }}$ switching is not sharply defined but rather broadly ranged around $10 \mathrm{~V}$ $\left(\sim 0.67 \mathrm{MV} \mathrm{cm}^{-1}\right)$, in agreement with the $P-E$ loop.

To ensure that the contrasts observed in dark-field TEM images are indeed $180^{\circ}$ head-to-head domains, we obtained atomic resolution high-angle annular dark-field (HAADF) scanning TEM (STEM) images after further thinning the TEM sample using low-energy $(900 \mathrm{eV})$ Ar-ion milling. As shown in Fig. 2g, a triangular domain is clearly visible and the $\mathrm{Ti} / \mathrm{Zr}$ columns are displaced in the opposite direction along the $c$ axis across the domain wall. For Ti-rich PZT thin films, the polarization direction is antiparallel with the $\mathrm{Ti} / \mathrm{Zr}$ displacement direction with respect to the $\mathrm{Pb}$ latttice ${ }^{13,16}$. We attribute the development of triangular domain shapes to anisotropy of domain growth ${ }^{3}$. Considering two components of domain wall speed for a given electric field, $v_{a}$ and $v_{c}$, along the $a$ axis and $c$ axis, respectively, we estimate that $v_{c}$ is approximately two to three times faster than $v_{a}$ based on the domain shapes in Fig. 2. It should be noted that both domain wall pinning by defects and anisotropic domain growth can be ruled out as an origin for the asymmetric domain switching and nonswitchable $P_{\text {up }}$ domains near the bottom interface. Especially, domain wall pinning can be easily overcome by applying a larger field. Recently, Gao et al. ${ }^{24}$ reported that the pinning force of misfit dislocations on domain wall motion is rather weak and slows down domain wall speed slightly. The domain wall pinning at defects plays some important roles in domain switching. However, the focus of this study is on the origins of the asymmetric switching and the nonswitchable $P_{\text {up }}$ domains near the PZT/Nb-STO interface.

Electric field mapping. In order to understand the origin of the nonswitchable interface polarization, we have mapped out electrostatic potentials and electric field distributions in PZT thin films under biased conditions using two different techniques: off-axis electron holography (Fig. 3) and EBIC measurements (Fig. 4). Electrostatic potential distributions were determined via electron-wave phase retrieval with various external biases, as shown in Fig. 3a-c. Electric potentials in the Nb-STO substrate increases with $+15 \mathrm{~V}$ compared with that in PZT film and vice versa for $-15 \mathrm{~V}$, clearly showing local potential changes because of application of external biases. However, any potential changes associated with the charged domain walls or depolarization field within domain are not detected in the unbiased $(0 \mathrm{~V})$ phase map (Fig. 3a), possibly because of effective compensation of the polarization charges at domain walls by mobile charge carriers or insufficient phase sensitivity of electron holography. In addition, no significant built-in potential across the PZT/Nb-STO interface is observed in the reconstructed phase image under $0 \mathrm{~V}$ biasing.
In our experiment, to avoid diffraction contrast related with dynamic variation of external biases resulted from the converse piezoelectric effect and non-uniform sample thickness, we extract the changes in electrostatic potential distributions solely caused by external biases by taking differences between the phase shifts obtained under external bias conditions and that under $0 \mathrm{~V}$ (see Methods section). The line profiles in Fig. $3 \mathrm{~d}$ show a considerable deviation from a linear potential distribution that one would expect for a pure dielectric assumption where no mobile charge carriers are considered. Especially, a noticeable potential barrier develops at the PZT/Nb-STO interface and increases as the positive bias applied to $\mathrm{Nb}-\mathrm{STO}$ is increased. In addition, distribution of electric fields along the $c$ axis (Fig. 3e), obtained by taking the first derivative of potential line profiles (Fig. $3 \mathrm{~d}$ ), shows that the electric fields are concentrated near the PZT/Nb-STO interface under positive biases. Here the positive sign of the electric field points from the bottom electrode to the top electrode. When negative biases are applied to $\mathrm{Nb}-\mathrm{STO}$, nearly no potential barriers and potential slopes are found at the PZT/NbSTO interface (blue lines in Fig. 3e). This unidirectional bias field near the PZT/Nb-STO interface can yield only $P_{\text {up }}$ domains, not $P_{\text {down }}$ domains, because applying negative bias to the substrate generates insufficient fields to initiate domain switching from $P_{\text {up }}$ to $P_{\text {down }}$. As a consequence, $P_{\text {up }}$ domains near the PZT/Nb-STO interface are unswitchable, in agreement with domain switching behaviour observed in Fig. 2.

We also measured EBIC signals under various external biases to further investigate electric field distributions. Total electric currents flowing through the sample have been collected as an analogue signal for each electron-beam position during electron beam scanning with dwell time of $32 \mu$ s during the STEMHAADF image acquisition, as shown in Fig. 4a. When a $200-\mathrm{keV}$ electron beam passes through the sample, it excites core level and valence electrons to conduction bands (CBs), generating electron-hole pairs (ehps). In the absence of electric field, these ehps diffuse out before they eventually recombine, yielding no net current. However, nonzero local electric fields can separate these ehps, generating an additional current $\left(I_{\mathrm{EBIC}}\right)$. Since ehp separation occurs more effectively under higher electric fields, $I_{\mathrm{EBIC}}$ is proportional to local electric field intensities smoothed by excess carrier diffusion. Previously, in the case of Si TEM samples, we have shown that the excess carrier diffusion length can be significantly reduced because of the enhanced surface recombinations ${ }^{28}$. We note that the EBIC signals collected with negative biases on Nb-STO were very noisy because of large forward currents flowing through the sample (Fig. 1b). Figure $4 \mathrm{~b}-\mathrm{e}$ shows a series of simultaneously acquired STEM-EBIC and STEMHAADF images under various positive biases. Although EBIC images are noisy because of the dynamic scanning noise of the electron beam, we can clearly see a bright band developing near the $\mathrm{PZT} / \mathrm{Nb}-\mathrm{STO}$ interface with increasing positive applied biases. This bright band suggests that large electric fields are indeed induced at the heterojunction with positive biasing, agreeing with the electric fields mapped out by electron holography (Fig. 3e). Under zero bias, there is a small and broad EBIC signal near the interface suggesting nonzero electric field associated with the band bending across the interface. Interestingly, EBIC signals expand more rapidly in the $\mathrm{Nb}-\mathrm{STO}$ side with increasing positive biases than in the PZT film. EBIC signals in PZT film are confined to $\sim 40 \mathrm{~nm}$ near the interface and are independent of bias voltage. This behaviour is also observed in potential profiles (Fig. 3d) obtained by off-axis electron holography. We attribute this to the presence of a higher concentration of deep levels continuously distributed throughout the band gap of PZT film, especially near the heterojunction due to large amount of structural defects associated with the lattice 

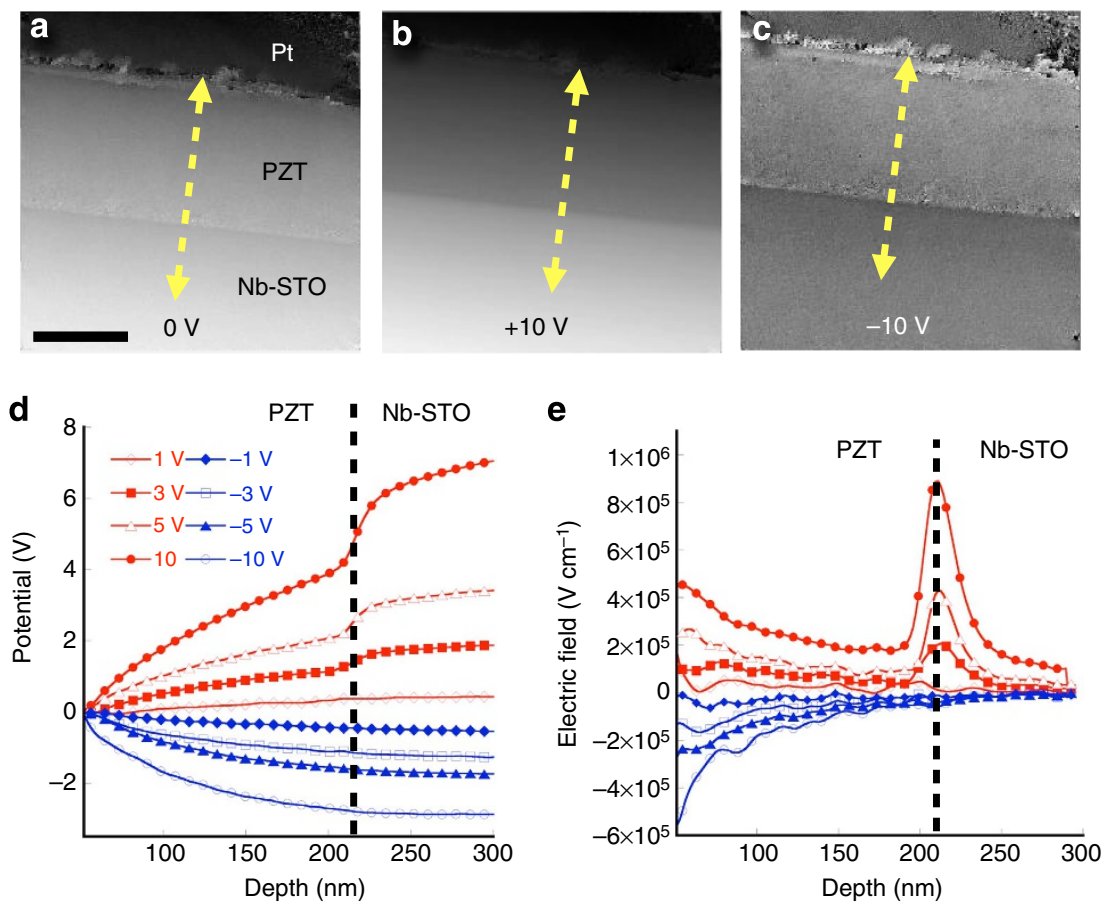

$\mathbf{f}_{E_{\mathrm{C}}}$

g

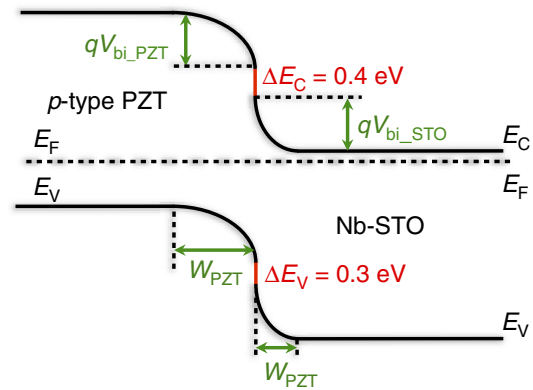

Ferroelectric PZT (RT)

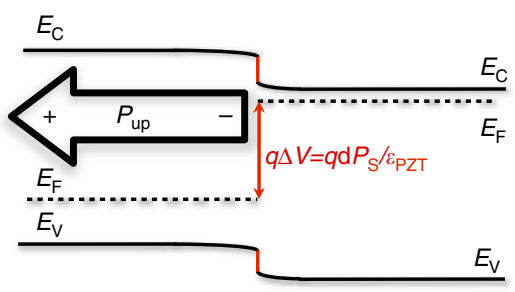

$E_{\mathrm{v}}$

Figure 3 | Electrostatic potential and electric field mapping using off-axis electron holography. (a-c) Reconstructed phase images with external biases of $0(\mathbf{a}),+15(\mathbf{b})$ and $-15 \mathrm{~V}(\mathbf{c})$, respectively, taken on the same area of PZT thin film. The scale bar is $100 \mathrm{~nm}$. (d,e) Line profiles of electrostatic potentials (d) and electric fields (e) quantified for the areas indicated in a-c with arrows. Potentials and fields shown here were taken with respect to $0-\mathrm{V}$ phase image (See Methods section). The potential at the Au/PZT interface has been chosen as a reference ( $0 \mathrm{~V}$ or electrical ground).

$(\mathbf{f}, \mathbf{g})$ Schematics of band bending across PZT/Nb-STO in paraelectric (f) and ferroelectric states $(\mathbf{g})$ of PZT films. Note that negative polarization charges near the interface suppress the band bending.

mismatch at the interface. These deep levels are perhaps mostly acceptor-like and trap electrons. In addition, converse piezoelectric effect of the PZT film may induce additional polarization charges with increasing biases.

The observed nonlinear potential profiles (that is, inhomogeneous electric fields) and unidirectional bias fields, consistently observed by both off-axis electron holography and EBIC measurements, can be explained if we consider PZT and $\mathrm{Nb}$-STO as wide band gap semiconductors that have electronic band bending across the heterojunction. According to the electron affinity rule, there is a type II band alignment across the PZT/Nb-STO heterojunction with $0.4 \mathrm{eV} \mathrm{CB}$ offset and $0.3 \mathrm{eV}$ valence band offset ${ }^{35}$, as shown in Fig. 3f. The electron concentration in $0.5 \mathrm{wt} \% \mathrm{Nb}$-doped STO is $\sim 2.2 \times 10^{20} \mathrm{~cm}^{-3}$, which pushes the Fermi level of Nb-STO close to the edge of the $\mathrm{CB}$ edge ${ }^{36}$. The PZT film behaves as a highly compensated semiconductor because of $\mathrm{Pb}$ and $\mathrm{O}$ vacancies, as well as deep levels associated with large amounts of structural defects because of the lattice mismatch ${ }^{37,38}$. In this work, we assume PZT is a $p$-type conductor with effective dopant concentration $\left(N_{\text {eff }}\right)$ of $\sim 10^{20} \mathrm{~cm}^{-3}$ considering acceptor-type deep levels and shallow doping levels ${ }^{39}$. Without considering local polarization charges, 1.9-V built-in potential is drawn for the heterojunction between PZT thin film and Nb-STO substrate, as shown in Fig. 3f. According to the heterojunction band alignment theory ${ }^{40}$, the potential drops ( $V_{\mathrm{PZT}}$ and $\left.V_{\mathrm{STO}}\right)$ and depletion region widths ( $W_{\text {PZT }}$ and $W_{\text {STO }}$ ) supported by each PZT and Nb-STO under thermal equilibrium and the depletion approximation can be determined as:

$$
\begin{gathered}
V_{\mathrm{PZT}}=\frac{\varepsilon_{\mathrm{STO}} N_{\mathrm{D}}}{\varepsilon_{\mathrm{STO}} N_{\mathrm{D}}+\varepsilon_{\mathrm{PZT}} N_{\mathrm{eff}}}\left(V_{\mathrm{bi}}+V\right) \\
V_{\mathrm{STO}}=\frac{\varepsilon_{\mathrm{PZT}} N_{\mathrm{eff}}}{\varepsilon_{\mathrm{STO}} N_{\mathrm{D}}+\varepsilon_{\mathrm{PZT}} N_{\mathrm{eff}}}\left(V_{\mathrm{bi}}+V\right) \\
W_{\mathrm{PZT}}=\sqrt{\frac{2 \varepsilon_{\mathrm{PZT}} \varepsilon_{\mathrm{STO}} N_{\mathrm{D}}\left(V_{\mathrm{bi}}+V\right)}{q N_{\mathrm{eff}}\left(\varepsilon_{\mathrm{STO}} N_{\mathrm{D}}+\varepsilon_{\mathrm{PZT}} N_{\mathrm{eff}}\right)}} \\
W_{\mathrm{STO}}=\sqrt{\frac{2 \varepsilon_{\mathrm{PZT}} \varepsilon_{\mathrm{STO}} N_{\mathrm{eff}}\left(V_{\mathrm{bi}}+V\right)}{q N_{\mathrm{D}}\left(\varepsilon_{\mathrm{STO}} N_{\mathrm{D}}+\varepsilon_{\mathrm{PZT}} N_{\mathrm{eff}}\right)}}
\end{gathered}
$$


a
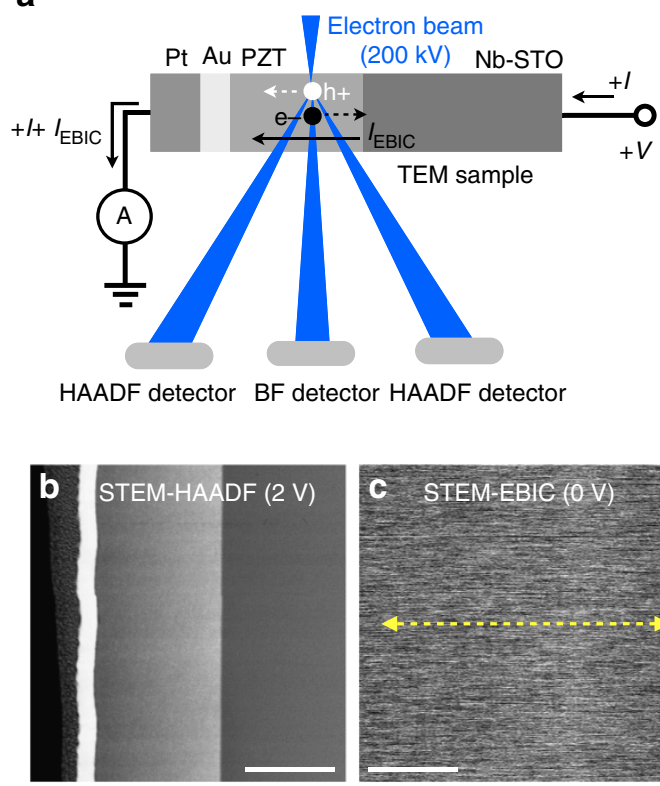
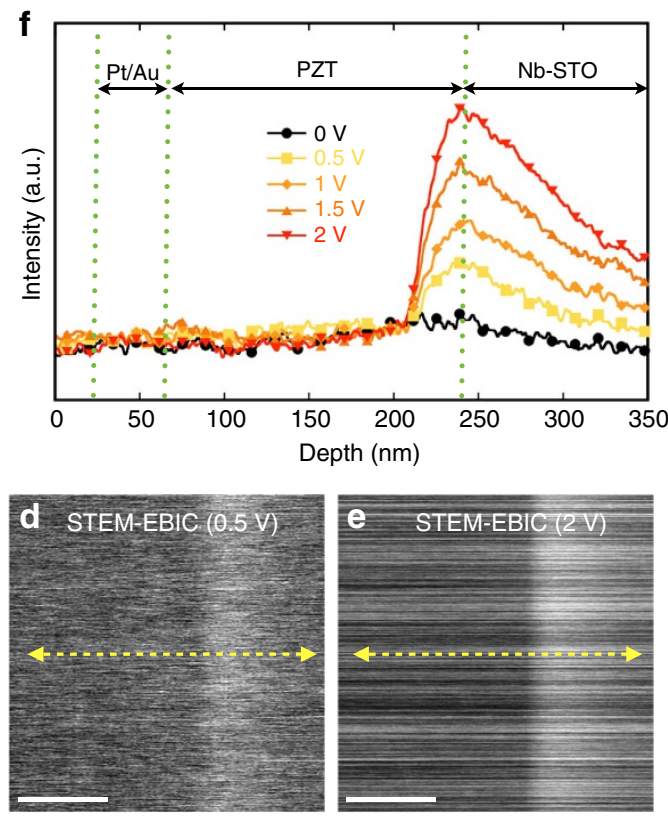

Figure 4 | Mapping electron-beam-induced current. (a) A schematic of simultaneous acquisition of STEM-HAADF and STEM-EBIC images. (b) A STEM-HAADF image taken with $2 \mathrm{~V}$ on $\mathrm{Nb}$-STO substrate. (c-e) STEM-EBIC images taken with $0,0.5$ and $2 \mathrm{~V}$, respectively. b,e were acquired simultaneously. All scale bars are $50 \mathrm{~nm}$. (f) Line profiles of STEM-EBIC signals from the areas indicated in c-e.

where $V_{\mathrm{bi}}$ is the built-in potential, $\varepsilon_{\mathrm{PZT}}$ and $\varepsilon_{\mathrm{STO}}$ dielectric constants for PZT and $\mathrm{Nb}-\mathrm{STO}$, respectively, $N_{\mathrm{D}}$ dopant concentration in $\mathrm{Nb}$-STO, $q$ electronic charge and $V$ reverse bias. An estimate of each built-in potentials and depletion regions can be made if the dielectric constants of PZT and Nb-STO are known. Inserting $\varepsilon_{\mathrm{PZT}}=200 \varepsilon_{0}$ (ref. 39) and $\varepsilon_{\mathrm{STO}}=50 \varepsilon_{0}$ (ref. 41) into the above equations gives $V_{\text {bi_PZT }}=0.67 \mathrm{~V}$ and $V_{\text {bi_STO }}=1.23 \mathrm{~V}$, and $W_{\mathrm{PZT}}=12 \mathrm{~nm}$ and $W_{\mathrm{STO}}=6 \mathrm{~nm}$, respectively, under zero bias. The charge neutrality condition is met by $q W_{\mathrm{PZT}} N_{\mathrm{eff}}=q W_{\mathrm{STO}} N_{\mathrm{D}} \sim 19.2 \mu \mathrm{C} \mathrm{cm}{ }^{-2}$.

The PZT/Nb-STO heterojunction is therefore expected to exhibit a rectifying property since positive bias or negative bias applied to Nb-STO will increase or decrease, respectively, the energy barrier for an electron injection from the Nb-STO. Under reverse (positive) bias, most of the applied voltage is drawn across the reverse-biased PZT/Nb-STO, while under forward (negative) bias, negligibly small voltage across the forward-biased $\mathrm{PZT} / \mathrm{Nb}$ STO, being consistent with electron holography (Fig. 3) and EBIC results (Fig. 4). Linear potential profiles near the top interface for both positive and negative biases in Fig. 3 suggest Ohmic-like behaviour of the top interface. This Ohmic behaviour can be attributed to high density of interface states due to extended exposure of PZT film surface to atmosphere after film deposition and room-temperature top electrode $(\mathrm{Au})$ deposition. Conduction across the top electrode/PZT interface occurs through these interface states, not by band bending. As a consequence, electric transport is mainly governed by minority carrier (electron) injection across the PZT/Nb-STO interface, leading to a rectifying behaviour.

The polarization charge near the interface will influence the zero-bias band bending and the associated built-in potential. Conceptually, polarization charge can be treated as an equivalent to an external bias to induce the same amount of charge within the PZT film ${ }^{42}$. The presence of negative polarization charge of $P_{\text {up }}$ domain near the PZT/Nb-STO interface can be considered as a forward biasing the heterojunction (negative bias to $\mathrm{Nb}-\mathrm{STO}$ ), inducing the same amount of charge as the spontaneous polarization $\left(P_{\mathrm{S}}\right)$. Consequently, the negative polarization charge of the $P_{\text {up }}$ domain counteracts total space charges on both sides of the heterojunction. The sum of total space charge densities stored in both depletion regions in the band diagram shown in Fig. 3f under zero bias is $38.4 \mu \mathrm{Ccm}^{-2}$, which is comparable to typical polarization charges reported for epitaxial PZT thin films. Another simple way to estimate influence of polarization charges on band bending is using the dielectric gap model $^{39}$ that considers polarization charge as a sheet charge separated from the interface by a dielectric gap to give rise to a change in built-in potential, $\Delta V=\frac{P_{\mathrm{S}}}{\varepsilon_{\mathrm{PZT}}} d$. Here $\Delta V$ is a change in built-in potential because of presence of polarization charge located from the heterojunction by a gap $(d)$. Estimation of $\Delta V$ requires knowledge of exact values of $P_{\mathrm{S}}, \varepsilon_{\mathrm{PZT}}$ and $d$. For an epitaxial PZT film, it is reported that the band bending modulation because of polarization charge can be of the order of $0.71 \mathrm{eV}^{43}$ using 150 as the PZT dielectric permittivity, $1 \mathrm{~nm}$ gap and $95 \mu \mathrm{Ccm}^{-2}$ of polarization charge. We note that the dielectric constant in the gap within the PZT film near the interface can be significantly lower than that of intrinsic PZT. This would significantly increase the band bending modulation. Interestingly, a dielectric permittivity of $\sim 50$ for the gap layer results in a flat band condition across the PZT/Nb-STO heterojunction (exactly cancelling out the built-in potential of $1.9 \mathrm{eV}$ ) if we assume $80 \mu \mathrm{Ccm}^{-2}$ polarization charge for PZT film and 1-nm dielectric gap. This 'flat-band' scenario (Fig. $3 \mathrm{~g}$ ) is supported by off-axis electron holography and EBIC data that show no measurable band bending or small $I_{\mathrm{EBIC}}$ across the PZT/ $\mathrm{Nb}$-STO interface under zero-bias condition, as shown in Figs 3 and 4.

Origin of the charged domain wall formation. One scenario leading to the formation of head-to-head domain walls in asgrown PZT thin films is as follows. We assume that the PZT films were grown in the paraelectric phase because of the high deposition temperature of $515^{\circ} \mathrm{C}$. Owing to the band bending across the PZT/Nb-STO heterojunction, a built-in electric field pointing from $\mathrm{Nb}-\mathrm{STO}$ to $\mathrm{PZT}$ will be induced within the 
a

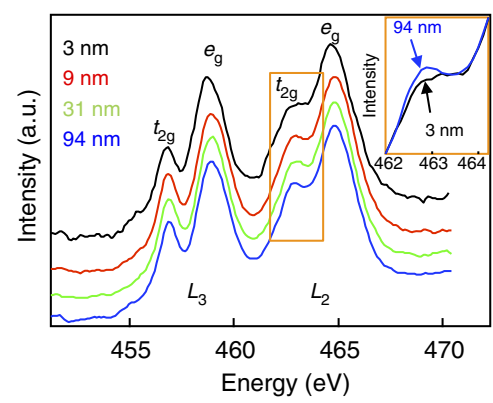

b

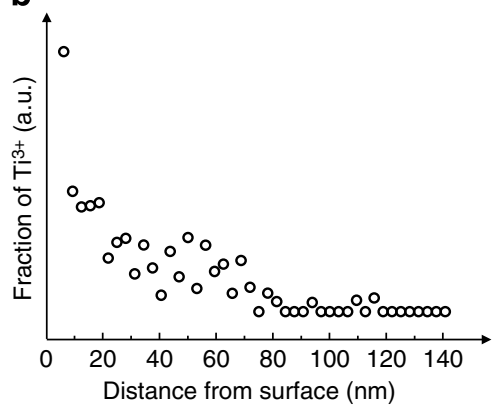

C

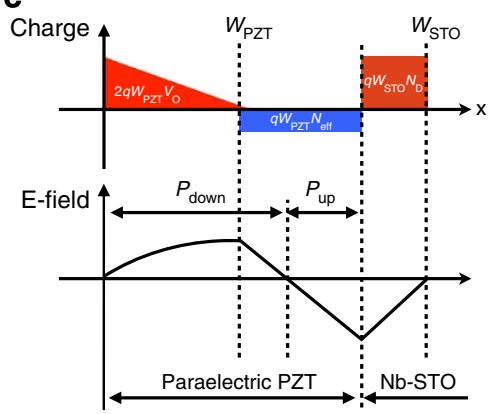

Figure 5 | Progress transition of Ti valence states. (a) EELS spectra of Ti-L edges for different positions in PZT film. A 2D spectrum image (22 $\times 63$ pixels) was acquired with $3.1 \mathrm{~nm}$ pixel width from a rectangular region. Lateral (in plane direction) 22 spectra were summed to enhance signal-to-noise ratio. Each number indicates the distance in $\mathrm{nm}$ from the surface of the PZT film. (b) Multiple least-square fitting results with the $94 \mathrm{~nm}$ spectrum as a reference. (c) A schematic of charge (top) and electric field (bottom) distributions in paraelectric PZT film. $V_{O}$ represents the concentration of doubly charged oxygen vacancy.

depletion region. When the PZT film goes through the paraelectric-ferroelectric phase transition upon cooling, the $P_{\text {up }}$ domain will be induced near the interface because its polarization direction is parallel to the built-in electric field within the PZT depletion region. However, near the surface of the PZT film depending on local chemistry and defect natures, opposite builtin electric field can be present. We investigated local Ti valence changes in the PZT thin films using electron-energy loss spectroscopy (EELS). Figure 5a shows a series of Ti-L edges from several positions in the PZT film as a function of distance from the top interface. A systematic trend, especially regarding the change of the $\mathrm{Ti} \mathrm{L}_{2}$ peak intensities, is observed when comparing with the $\mathrm{Ti} \mathrm{L}_{2}$ edge taken from $94 \mathrm{~nm}$ away from the top interface. The $\mathrm{Ti} \mathrm{L}_{2}$ edge taken near the top interface ( $3 \mathrm{~nm}$ away from the surface) shows two main constituent peaks $\left(t_{2 \mathrm{~g}}\right.$ and $\left.e_{\mathrm{g}}\right)$ develop noticeable shoulders, as shown in the inset of Fig. 5a, in agreement with trends reported in the literature ${ }^{44}$. Using the Ti- $\mathrm{L}$ edge far from the surface ( $94 \mathrm{~nm}$ away) as a reference, we plotted the differences in Ti-L edges using a multiple least-square fitting (Fig. 5b). This shows a progressive transition in the relative $\mathrm{Ti}$ valence state towards the surface of the PZT film. Although the exact valence state of $\mathrm{Ti}$ at the surface is difficult to quantify, the subtle change of Ti valence suggests more conduction electrons near the surface of PZT film, implying the presence of oxygen vacancy in the PZT film. A higher concentration of oxygen vacancy near the surface of PZT film will generate a net electric field pointing from the surface to the film/substrate interface, inducing $P_{\text {down }}$ domains near the surface region of PZT, despite of the energy cost for the formation of head-to-head $180^{\circ}$ domain walls. Mobile electrons from near-surface oxygen vacancies will migrate and accumulate around the charged domain walls for its stabilization via internal charge screening. Mokry et al. ${ }^{45}$ reported in their theoretical study that free charge compensation of bound charge can lead to a strong reduction in the pressure exerted on charged domain wall from an external electric field. This supports the scenario described here, of bound charge compensation by mobile charge carriers for their stabilization. A schematic for charge distributions and built-in electric fields in paraelectric phase of the PZT film is shown in Fig. 5c. This scenario is similar to a back-to-back Schottky diode model for metal-ferroelectric-metal structures $^{39}$, where two opposite built-in electric fields pointing at each other are set from the top and bottom electrode interfaces.

\section{Discussion}

Our study reveals that the built-in electric fields associated with electronic band bending across the $\mathrm{PZT} / \mathrm{Nb}-\mathrm{STO}$ heterojunction and higher oxygen vacancy concentration near the surface of the PZT film induce $P_{\text {up }}$ and $P_{\text {down }}$ domains, respectively, upon paraelectric-ferroelectric phase transition, which counteract each built-in electric field. The heterojunction behaves as a $p n$ junction and produces unidirectional bias fields, prohibiting domain switching of $P_{\text {up }}$ domains near the heterojunction. As a result, energetically unfavourable charged domain walls with head-tohead configuration are induced in the middle of PZT films during domain switching process initiated by external biasing. These charged domain walls are found to be stable because of internal charge compensation by mobile charge carriers in the PZT film. Our observation of nonswitchable $P_{\text {up }}$ domains near the PZT/NbSTO interface and formation of charged domain walls in the middle of the PZT film can be an origin of imprint (preferred $P_{\text {up }}$ $\left(P_{\text {down }}\right)$ domains near the bottom (top) interface) and retention loss (possible backswitching near the top and bottom interfaces) in ferroelectric thin films. We also point out that the switchable diode effects have not been previously observed for PZT thin films ${ }^{42}$, in contrary to $\mathrm{BiFeO}_{3}$ (ref. 46). Our results imply that a nonswitchable $P_{\text {up }}$ domain near the bottom interface can be the origin for the absence of band bending modulation by polarization charge in the PZT/Nb-STO system.

\section{Methods}

Thin film growth and characterization. Thin films of PZT are grown on $5 \times$ $5 \times 0.5 \mathrm{~mm}$ samples of $0.5 \mathrm{wt}$.\% Nb-STO (001) obtained from Crystec. The films of PZT are grown using off-axis radiofrequency magnetron sputtering, under flowing $\operatorname{Ar}(33.7 \mathrm{sccm})$ and $\mathrm{O}_{2}(13.7 \mathrm{sccm})$, where $\mathrm{sccm}$ refers to the cubic centimetres per minute at standard temperature and pressure. The chamber pressure is maintained at $225 \mathrm{mTorr}$ and the substrate temperature is $515^{\circ} \mathrm{C}$. The deposition rate is calibrated using X-ray reflectivity and is $\sim 4.5 \AA \mathrm{min}^{-1}$. The samples are cooled in the process gases at a chamber pressure of 225 mTorr. The thickness of films analysed here are $\sim 150 \mathrm{~nm}$. X-ray diffraction scans (Supplementary Fig. 1) demonstrate the growth of high-quality single crystalline films of PZT. The (001) rocking curve (Supplementary Fig. 1c) has a full-width half maximum of $0.04^{\circ}$, the same as the Nb-STO substrate.

PFM. PFM was performed by writing squares with a DC bias voltage of $\pm 10 \mathrm{~V}$. Readout was performed with an AC-excitation voltage of $1 \mathrm{~V}$, at a frequency far off resonance. $P-E$ loop measurement was performed with an aixACCT ferroelectric tester on a PZT thin film sample using a pulsed positive up negative down measurement (150 nm PZT on Nb-STO (001)). The pulsed measurement facilitates subtraction of the leakage current, leaving only the switching current.

TEM. Final FIB milling for TEM sample preparation was carried out using $5 \mathrm{keV}$ $\mathrm{Ga}^{+}$ions to minimize ion-beam-induced damages. For STEM-HAADF, the sample was further milled with $900 \mathrm{eV}$ Ar ions with $\pm 10^{\circ}$ incidence angles using Nanomill (Fischione Instruments Inc.). A JEOL 2100F Lorentz microscope was used for off-axis electron holography. A JEOL ARM 200CF equipped with a cold field-emission gun and double-spherical aberration correctors at the Brookhaven National Laboratory operated at $200 \mathrm{kV}$ was used for STEM-HAADF. The collection angles for HAADF detectors were from 68 to $280 \mathrm{mrad}$. EBIC signals were acquired simultaneously with STEM-HAADF images with the preamplifier installed with holder controller provided by Nanofactory Instruments AB. EELS data were obtained with $0.1 \mathrm{eV} / \mathrm{ch}$ dispersion. 
Data analysis of electron holograms. The electrostatic potential distributions across top electrode/PZT/Nb-STO under external biases were mapped out by off-axis electron holography. Phase shift $\phi(x)_{O V}$ in imaging $200 \mathrm{keV}$ electron wave measured using electron holography under zero external bias condition is quantitatively related to electrostatic potentials within the sample and local thickness $t(x)$ in $(\mathrm{nm})$ as the following;

$$
\varphi(x)_{0 V}=C_{\mathrm{E}}\left[V_{\mathrm{MIP}}(x)+V_{\mathrm{bi}}(x)\right] t(x)
$$

where $C_{\mathrm{E}}$ is a constant of $0.00729 \mathrm{radV}^{-1} \cdot \mathrm{nm}$ for $200 \mathrm{keV}$ electron beam, $V_{\mathrm{MIP}}$ $(x)$ the mean inner potential in $(\mathrm{V})$ and $V_{\mathrm{bi}}(x)$ the built-in potential in $(\mathrm{V})$. Here the $x$ axis is taken along the $c$ axis and no fringing fields outside TEM samples are assumed. $V_{\text {MIP }}(x)$ is the mean inner potential that the imaging electron would see in the crystal and differs for PZT film and Nb-STO substrate. If we neglect unit-cell volume changes under biased conditions because of converse piezoelectric effects of PZT thin films, then $V_{\mathrm{MIP}}(x)$ can be treated as a constant. $V_{\mathrm{bi}}(x)$ is associated both with the depolarization fields because of uncompensated polarization charges in PZT thin films and the built-in electric fields across top and bottom interfaces because of electronic band bending to reach a electrochemical equilibrium. If we assume the full polarization charge compensation at the top interface, then $V_{\mathrm{bi}}(x)$ across the PZT film is mainly attributed to the electronic band bending across the PZT/Nb-STO heterojunction as a result of charge exchange considering the effects of polarization charge. We estimate that $V_{\mathrm{bi}}(x)$ at $0 \mathrm{~V}$ is small for $P_{\mathrm{up}}$ domain in PZT film from EBIC (Fig. 4) because the EBIC is proportional to charge collection volumes in the sample, that is, product of depletion region width and thickness of the sample. Thus, phase shift measured by electron holography at $0 \mathrm{~V}$ mainly shows the contribution due to the product of $V_{\mathrm{MIP}}(x)$ and local thickness $t(x)$. Under external biases, the term $V_{\text {ext }}(x)$ describes electrostatic potential distributions due to external biases on the Nb-STO substrate.

$$
\varphi_{ \pm V}(x)=C_{\mathrm{E}}\left[V_{\mathrm{MIP}}(x)+V_{\mathrm{bi}}(x) \pm V_{\text {ext }}(x)\right] t(x)
$$

In a pure dielectric assumption where no mobile charge carriers are considered, application of external bias results in constant electric fields and thus linearly changing electrostatic potentials $V_{\text {ext }}(x)$ across PZT thin films. However, when there are mobile carriers in the PZT thin film and interface dipoles due to formation of PZT/Nb-STO heterojunction in an electrochemical equilibrium, electric fields due to external biases will be more concentrated around top or bottom interfaces due to corresponding electronic band bending. We extract the changes in electrostatic potential distributions solely caused by external biases $\left(V_{\text {ext }}(x)\right)$ by taking differences between the phase shifts obtained under external bias conditions and that under $0 \mathrm{~V}$ as the followings:

$$
\begin{gathered}
\Delta \varphi_{ \pm V}(x)=\varphi_{ \pm V}(x)-\varphi_{0 V}(x)=C_{E}\left[ \pm V_{\text {ext }}(x)\right] t(x) \\
\pm V_{\text {ext }}(x)=\frac{\Delta \varphi_{ \pm V}(x)}{C_{\mathrm{E}} t(x)}
\end{gathered}
$$

We estimated the local sample thickness $t(x)$ in the reconstructed amplitude images from electron holograms ${ }^{47}$. The sample thickness is found to be linearly increasing towards the substrate (Supplementary Fig. 2). To calibrate thickness from amplitude images, we used $115 \mathrm{~nm}$ as the mean free path for inelastic scattering to find thickness profile in Nb-STO region ${ }^{48}$. The determined thickness profile was extended towards the PZT film to obtain whole thickness profile across PZT thin film/Nb-STO substrate to obtain the line profiles of $V_{\text {ext }}(x)$ using the equation (8).

\section{References}

1. Catalan, G., Seidel, J., Ramesh, R. \& Scott, J. Domain wall nanoelectronics. Rev. Mod. Phys. 84, 119-156 (2012).

2. Ahn, C. H., Triscone, J. M. \& Mannhart, J. Electric field effect in correlated oxide systems. Nature 424, 1015-1018 (2003).

3. Dawber, M., Rabe, K. M. \& Scott, J. F. Physics of thin-film ferroelectric oxides. Rev. Mod. Phys. 77, 1083-1130 (2005).

4. Setter, N. et al. Ferroelectric thin films: review of materials, properties, and applications. J. Appl. Phys. 100, 051606 (2006).

5. Warren, W. L. et al. Voltage shifts and imprint in ferroelectric capacitors. Appl. Phys. Lett. 67, 866-868 (1995)

6. Warren, W. L., Tuttle, B. A. \& Dimos, D. Ferroelectric fatigue in perovskite oxides. Appl. Phys. Lett. 67, 1426-1428 (1995).

7. Damjanovic, D. Ferroelectric, dielectric and piezoelectric properties of ferroelectric thin films and ceramics. Rep. Prog. Phys. 61, 1267-1324 (1998).

8. Choi, K. J. et al. Enhancement of ferroelectricity in strained $\mathrm{BaTiO}_{3}$ thin films. Science 306, 1005-1009 (2004).

9. Ederer, C. \& Spaldin, N. A. Effect of epitaxial strain on the spontaneous polarization of thin film ferroelectrics. Phys. Rev. Lett. 95, 257601 (2005).

10. Schlom, D. G. et al. Strain tuning of ferroelectric thin films. Annu. Rev. Mater. Res. 37, 589-626 (2007).

11. Pertsev, N. A., Zembilgotov, A. G. \& Tagantsev, A. K. Effect of mechanical boundary conditions on phase diagrams of epitaxial ferroelectric thin films. Phys. Rev. Lett. 80, 1988-1991 (1998).
12. Gerra, G., Tagantsev, A. K., Setter, N. \& Parlinski, K. Ionic polarizability of conductive metal oxides and critical thickness for ferroelectricity in $\mathrm{BaTiO}_{3}$. Phys. Rev. Lett. 96, 107603 (2006).

13. Chisholm, M. F., Luo, W. D., Oxley, M. P., Pantelides, S. T. \& Lee, H. N. Atomic-scale compensation phenomena at polar interfaces. Phys. Rev. Lett. 105, 197602 (2010).

14. Streiffer, S. K. et al. Observation of nanoscale 180 degrees stripe domains in ferroelectric $\mathrm{PbTiO}_{3}$ thin films. Phys. Rev. Lett. 89, 067601 (2002).

15. Nelson, C. T. et al. Spontaneous vortex nanodomain arrays at ferroelectric heterointerfaces. Nano Lett. 11, 828-834 (2011).

16. Jia, C. L., Urban, K. W., Alexe, M., Hesse, D. \& Vrejoiu, I. Direct observation of continuous electric dipole rotation in flux-closure domains in ferroelectric $\mathrm{Pb}(\mathrm{Zr}, \mathrm{Ti}) \mathrm{O}_{3}$. Science 331, 1420-1423 (2011).

17. Xiao, Y., Shenoy, V. B. \& Bhattacharya, K. Depletion layers and domain walls in semiconducting ferroelectric thin films. Phys. Rev. Lett. 95, 247603 (2005).

18. Zubko, P., Jung, D. J. \& Scott, J. F. Space charge effects in ferroelectric thin films. J. Appl. Phys. 100, 114112 (2006).

19. Zhang, J., Tang, M. H. \& He, J. Doping concentration and thickness effects in ferroelectric thin films. Appl. Phys. Lett. 96, 122905 (2010).

20. Gureev, M. Y., Tagantsev, A. K. \& Setter, N. Head-to-head and tail-to-tail $180^{\circ}$ domain walls in an isolated ferroelectric. Phys. Rev. B 83, 184104 (2011).

21. Balke, N. et al. Direct observation of capacitor switching using planar electrodes. Adv. Funct. Mater. 20, 3466-3475 (2010).

22. Nelson, C. T. et al. Domain dynamics during ferroelectric switching. Science 334, 968-971 (2011).

23. Chang, H. J. et al. Watching domains grow: in-situ studies of polarization switching by combined scanning probe and scanning transmission electron microscopy. J. Appl. Phys. 110, 052014 (2011).

24. Gao, P. et al. Revealing the role of defects in ferroelectric switching with atomic resolution. Nat. Commun. 2, 1-6 (2011).

25. Winkler, C. R., Damodaran, A. R., Karthik, J., Martin, L. W. \& Taheri, M. L. Direct observation of ferroelectric domain switching in varying electric field regimes using in situ TEM. Micron 43, 1121-1126 (2012).

26. Han, M. G. et al. Ferroelectric switching dynamics of topological vortex domains in a hexagonal manganite. Adv. Mater. 25, 2415-2421 (2013).

27. Han, M. G., Smith, D. J. \& McCartney, M. R. In situ electron holographic analysis of biased Si n(+)-p junctions. Appl. Phys. Lett. 92, 143502 (2008).

28. Han, M. G. et al. Direct measurement of electron beam induced currents in p-type silicon. Solid State Electron 54, 777-780 (2010).

29. Blank, H. \& Amelinckx, S. Direct observation of ferroelectric domains in barium titanate by means of the electron microscope. Appl. Phys. Lett. 2, 140-142 (1963).

30. Tanaka, M. Acta Crystallogr. A A 31, 59 (1975).

31. Klein, L. J. et al. J. Vac. Sci. Technol. B 28, C5A20 (2010).

32. McQuaid, R. G. P., McMillen, M., Chang, L.-W., Gruverman, A. \& Gregg, J. M. Domain wall propagation in meso- and nanoscale ferroelectrics. J. Phys. Condens. Matter 24, 024204 (2012).

33. Chu, M.-W. et al. Impact of misfit dislocations on the polarization instability of epitaxial nanostructured ferroelectric perovskites. Nat. Mater. 3, 87-90 (2004).

34. Pintilie, L., Pintilie, I., Vrejoiu, I. \& Alexe, M. In 2008 International Semiconductor Conference, CAS 2008 Proceedings 2, 295-298 (2008).

35. Robertson, J. Band offsets of wide-band-gap oxides and implications for future electronic devices. J. Vac. Sci. Technol. B 18, 1785-1791 (2000).

36. Watanabe, Y. \& Okano, M. Photoresponse of Zener tunneling junctions of $\mathrm{Pb}(\mathrm{Ti}, \mathrm{Zr}) \mathrm{O}_{3} / \mathrm{SrTiO}_{3}$ at low temperature. J. Appl. Phys. 94, 7187-7192 (2003).

37. Gerson, R. \& Jaffe, H. Electrical condductivity in lead titanate zirconate ceramics. J. Phys. Chem. Solids 24, 979-984 (1963).

38. Pintilie, L., Vrejoiu, I., Le Rhun, G. \& Alexe, M. Short-circuit photocurrent in epitaxial lead zirconate-titanate thin films. J. Appl. Phys. 101, 064109 (2007).

39. Pintilie, L. \& Alexe, M. Metal-ferroelectric-metal heterostructures with Schottky contact: I. Influence of the ferroelectric properties. J. Appl. Phys. 98, 1242013 (2005).

40. Shur, M. Physics of Semiconductor Devices (Prentice-Hall Inc., 1990).

41. Ruotolo, A., Lam, C. Y., Cheng, W. F., Wong, K. H. \& Leung, C. W. High-quality all-oxide Schottky junctions fabricated on heavily doped $\mathrm{Nb}: \mathrm{SrTiO}_{3}$ substrates. Phys. Rev. B 76, 075122 (2007).

42. Tayebi, N. et al. Tuning the built-in electric field in ferroelectric $\mathrm{Pb}\left(\mathrm{Zr}_{0.2} \mathrm{Ti}_{0.8}\right) \mathrm{O}_{3}$ films for long-term stability of single-digit nanometer inverted domains. Nano Lett. 12, 5455-5463 (2012).

43. Hrib, L. M. et al. Electrode interface control of the Schottky diode-like behavio in epitaxial $\mathrm{Pb}\left(\mathrm{Zr}_{0.2} \mathrm{Ti}_{0.8}\right) \mathrm{O}_{3}$ thin films: a critical analysis. J. Appl. Phys. 113, 214108 (2013)

44. Shao, Y., Maunders, C., Rossouw, D., Kolodiazhnyi, T. \& Botton, G. A. Quantification of the Ti oxidation state in $\mathrm{BaTi}_{1}{ }_{\mathrm{x}} \mathrm{Nb}_{\mathrm{x}} \mathrm{O}_{3}$ compounds. Ultramicroscopy 110, 1014-1019 (2010).

45. Morky, P., Tagantsev, A. K. \& Fousek, J. Pressure on charged domain walls and additional imprint mechanism in ferroelectrics. Phys. Rev. B 75, 094110 (2007). 
46. Choi, T. et al. Switchable ferroelectric diode and photovoltaic effect in $\mathrm{BiFeO}_{3}$. Science 324, 63 (2009).

47. McCartney, M. R. \& Gajdardziskajosifovska, M. Absolute measurement of normalized thickness, $\mathrm{T} / \lambda(\mathrm{I})$, from off-axis electron holography. Ultramicroscopy 53, 283-289 (1994).

48. Egerton, R. F. Electron Energy-Loss Spectroscopy in the Electron Microscope (Springer, 1996).

\section{Acknowledgements}

R. Dunin-Borkowski and M. McCartney are acknowledged for their fruitful discussions on electron holography data. M. McCartney is also acknowledged for providing the Digital Micrograph script used in reconstruction of electron holograms. L.Q. Chen is acknowledged for helpful phase field simulations. TEM sample preparation in part was carried out by K. Kisslinger at the Center for Functional Nanomaterials, Brookhaven National Laboratory. Research carried out in part at the Center for Functional Nanomaterials, Brookhaven National Laboratory, which is supported by the U.S. Department of Energy, Office of Basic Energy Sciences. We acknowledge support from NSF MRSEC DMR 119826 (CRISP), DMR 1309868 and FAME. This work is supported by the U.S Department of Energy, Office of Basic Energy Science, Division of Materials Science and Engineering, under Contract number DE-AC02-98CH10886. Supplementary Information is available online.

\section{Author contributions}

M.-G.H., M.A.S., F.J.W., C.H.A. and Y.Z. conceived the research. M.S.J.M., J.H. and F.J.W. deposited epitaxial thin films. M.S.J.M. performed P-E, PFM and XRD measurements and analysed data with F.J.W. M.-G.H. and M.A.S. performed in situ domain switching experiments. M.-G.H. performed holography and analysed data. M.-G.H. and R.T. performed STEM-EBIC experiments. M.-G.H. and T.A. obtained STEM images and EELS data. L.W. analysed STEM images and EELS data. Y.Z., F.J.W. and C.H.A. directed research. M.-G.H. wrote the manuscript with Y.Z. All authors discussed and contributed to the manuscript.

\section{Additional information}

Supplementary Information accompanies this paper at http://www.nature.com/ naturecommunications

Competing financial interests: The authors declare no competing financial interests

Reprints and permission information is available online at http://npg.nature.com/ reprintsandpermissions/

How to cite this article: Han, M.-G. et al. Interface-induced nonswitchable domain in ferroelectric thin films. Nat. Commun. 5:4693 doi: 10.1038/ncomms5693 (2014) 